\title{
DESIGNING AND OPTIMIZATION OF NAPROXEN SODIUM DEFORMABLE VESICULAR SYSTEMS THROUGH FACTORIAL DESIGN: BOX BEHENKEN MODEL
}

\author{
NAVEEN GUPTA ${ }^{1 *}$, SHAILESH JAIN ${ }^{2}$ \\ 1,2School of Pharmacy, Madhyanchal Professional University, Bhopal, India \\ Email: naveenmpharm@gmail.com
}

Received: 28 Nov 2020, Revised and Accepted: 20 Jan 2021

\begin{abstract}
Objective: The objective of this investigation was to develop and statistically optimize deformable vesicles such as transfersomes and transethosomes of Naproxen sodium by employing $3^{3}$ factorial designs through software Design expert version 12 (Box-Behnken design) for dermal delivery.

Methods: The levels of the drug, phosphatidylcholine, and span 80 (independent variables) were varied to study the influence on vesicle size and \% entrapment efficiency (dependent variables) of transfersomes and for transethosomes, the levels of phosphatidylcholine, ethanol, and span 80 were selected as independent variables Second-order quadratic polynomial equation, 2D and 3D contour plots represented the relationship between variables and desired response. The optimization process was carried out using desirability plots and point prediction techniques.
\end{abstract}

Results: Results of the present study demonstrated that optimized transfersomes and transethosomes showed vesicle sizes of 114.91 nm and $102.91 \mathrm{~nm}$ respectively, while entrapment efficiency of $80.11 \%$ and $86.97 \%$, respectively. Both formulations showed high zeta potential values indicating the stability of the optimized formulation. ANOVA statistical results showed a significant difference $(\mathrm{P}<0.05)$

Conclusion: The results indicated that the independent variable plays a crucial role in optimizing a formulation that can be used for further research studies. Present preliminary study data provided strong evidence that the optimized deformable vesicular formulations through box Behnken factorial design can be a potentially useful drug carrier for naproxen sodium dermal delivery with minimum vesicle size and efficient entrapment efficiency.

Keywords: Naproxen sodium, Transfersomes, Transethosomes, Factorial design, Box Behnken design, Transdermal

(C) 2021 The Authors. Published by Innovare Academic Sciences Pvt Ltd. This is an open access article under the CC BY license (https://creativecommons.org/licenses/by/4.0/) DOI: https://dx.doi.org/10.22159/ijap.2021v13i2.40398. Journal homepage: https://innovareacademics.in/journals/index.php/ijap

\section{INTRODUCTION}

Rheumatoid arthritis is a major cause of disability, particularly in older individuals, the symptoms and signs of arthritis include pain, stiffness, swelling, muscle weakness, and limitation of movement of the joints [1]. Non-steroidal anti-inflammatory drugs like Naproxen sodium are an effective drug for the treatment of Arthritis. The antiinflammatory effect of NSAIDs is due to cyclooxygenase inhibition and the consequent reduction of prostaglandin synthesis leads to unfavorable side effects like GIT disorders, renal toxicity, hepatotoxicity, cardiovascular risks via systemic administration. So the urgent need is to develop a drug delivery system with enhanced target place localization and sustained drug release with minimum side effects. Designing a controlled and sustained release pattern of a drug relies on proper carrier selection, such as nanosome [2]. Several attempts have been made in the past to address this issue but each has its limitations. One strategy is topical delivery of drugs which has many advantages such as avoidance of hepatic first-pass metabolism, improved patient compliance, and ease of access [3]. Numerous approaches are utilized to enhance the effectiveness of the material for permeating through the skin such as penetration enhancers, iontophoresis, sonophoresis, etc. But due to the rigid skin barrier drugs have to face resistance in permeation also it shows an unpredictable drug release. Penetration enhancers and solvents irritation. The use of lipid vesicular carriers such as non-ionic surfactant-based niosomes and liposomes can overcome the filtration problem and penetrate the skin barrier along the transcutaneous moisture gradient [4].

The drawbacks associated with conventional carriers can be overcome by phospholipids-based colloidal drug delivery systems with high drug entrapment and penetration ability. These systems due to the biocompatible and biodegradable nature of phospholipids have gained much attention in recent times. The first such approach was the skin delivery of triamcinolone liposomes [5]. But fewer the flexibility of liposomal formulations restricts them to reach deeper layers of the skin $[6,7]$. Then Cevc introduced the concept of deformable vesicle transfersomes, which are capable of penetrating deeper into the skin by squeezing themselves; transfersomes are liposomes that contain edge activators including Tweens, span, sodium cholate, and sodium deoxycholate. These edge activators destabilize the bilayer lipid membrane of liposomes and increase the flexibility of vesicles to reach the deep inner dermal layer $[8,9]$

Ethosomes are vesicular carriers developed by Touito et al. [10], which consist of phospholipid, ethanol, and water. Ethosomes increase skin permeation due to the effect of ethanol on the lipid bilayer and increasing fluidity of skin stratum corneum lipid [11]. The transethosomes are composed of both ethanol and edge activators hence they have features of ethosomes and transfersomes which promise to be a better carrier for topical delivery. Incorporation in a gel system will enhance the permeation and leads to localization of the drug at the target site for desired sustained action with minimum side effects. and good rheological properties.

Various formulation factors play a crucial role in the formulation of these vesicles such as the concentration of phospholipid, edge activator, ethanol, drug amount, and many others. They directly affect the structure and efficiency of vesicles. The incorporation of an appropriate excipient in optimum concentration is a very important step in designing a formulation batch with respective therapeutic goals. This research focuses on the development and statistical optimization of vesicular carrier's formulation batches through experimental factorial design using screened factors and their levels which directly affect the therapeutic goals. For identifying the optimum formula, different independent variables that directly affect the properties of vesicles were studied by factorial design (Design Expert) software. The optimized formula by point prediction method is decided through experimental software. Then further experimentation and evaluation will be carried out in the next phase of research.

\section{MATERIALS AND METHODS}

\section{Materials}

Naproxen Sodium was received as a gift sample from Intas pharmaceuticals (Rangpo, Sikkim), Carbapol 940 was purchased 
from oxford laboratories, Mumbai, Span 80 was purchased from oxford laboratories, Egg Phosphatidylcholine was obtained from Doosan corporation (Korea) All of the solvents and chemicals were of the HPLC and analytical grades.

\section{Experimental methods}

\section{Method of preparation of naproxen sodium loaded transfersomes}

\section{Rotary evaporation-sonication method}

Egg Phosphatidylcholine and edge activator (surfactant) were dissolved in chloroform and methanol in a round bottom flask (RBF). The ratio of chloroform to methanol was $2: 1 \mathrm{v} / \mathrm{v}$. through rotary flash evaporator solvent evaporation results in the formation of a thin film. water is used as a hydrating medium for $1 \mathrm{~h}$ at room temperature for hydration of formed film, which resulted in multilamellar film vesicles. These vesicles are converted into small unilamellar vesicles through ultrasound cavitation and probe sonicator $[12,13]$.

Method of preparation of naproxen sodium loaded transethosomes

The cold method is used for preparing transethosomes, Egg Phosphatidylcholine was dissolved in ethanol in a conical flask with constant stirring at $700 \mathrm{rpm}$. The temperature of this alcoholic mixture was maintained at $30^{\circ} \mathrm{C}$. Drug and Span 80 were dissolved in water. This aqueous phase was then added to the alcoholic phase slowly in a fine stream with constant stirring at $700 \mathrm{rpm}$ in a closed vessel. It was stirred for an additional $5 \mathrm{~min}$. The system was kept at $30^{\circ} \mathrm{C}$ throughout the preparation. Size reduction was done by probe sonication for $5 \mathrm{~min}$ at RT. The selection of a method for the preparation of transethosomes was based on percent entrapment efficiency, stable transethosomal formulations can be achieved through the cold method [14].

\section{Experimental factorial design: box behenken}

\section{Optimization of process parameters variables}

The process of rotary vacuum evaporation were varied by varying the process variables to investigate their effect on the characteristics of carrier systems. The process variables Temperature and Rotation per minute (RPM) were varied based on 2 factors, 3 levels of general factorial design. and experimental trials were performed on all batches and based on the quality of film produced, the process was optimized $[15,16]$.

\section{Experimental design for the formulation of naproxen sodium loaded transfersomes}

The optimization of Naproxen Sodium Transfersomes was done by Design Expert, Version 12, Stat-Ease Inc. Minneapolis, USA) using three factors at three levels. It provides a rationale for understanding the possible interaction(s) among both independent and dependent variables and helps in selecting 'optimum' formulation in lesser experimental time. The design will help to identify the positive or negative effect of a different variable on desired responses. $\left(\mathrm{X}_{1}\right)$ Phosphatidylcholine $(\mathrm{mg})$ was taken as the first independent variable, $\left(\mathrm{X}_{2}\right)$ Span $80(\mathrm{mg})$ as the second independent variable and $\left(\mathrm{X}_{3}\right)$ Naproxen sodium $(\mathrm{mg})$ was selected as the third independent variables for Transfersomes. These variables varied at three levels, low level $(-1)$, medium level $(0)$, and high level $(+1)$. Vesicle size $(\mathrm{nm})\left(\mathrm{Y}_{1}\right)$ and $\%$ entrapment efficiency $\left(\mathrm{Y}_{2}\right)$ were selected as dependent (Response) variables [17].

Experimental design for the formulation of naproxen sodium loaded transethosomes

Phosphatidylcholine $(\mathrm{mg})\left(\mathrm{X}_{1}\right)$ was taken as the first independent variable, Ethanol \% $\left(\mathrm{X}_{2}\right)$ was selected as the second independent variable, and $\left(\mathrm{X}_{3}\right)$ Span $80(\mathrm{mg})$ as the third independent variables. These variables varied at three levels, low level $(-1)$, medium level $(0)$, and high level $(+1)$. The amount of drugs were kept constant. Vesicle size $(\mathrm{nm})\left(\mathrm{Y}_{1}\right)$ and \% entrapment efficiency $\left(\mathrm{Y}_{2}\right)$ were selected as dependent (Response) variables.

\section{Characterization of deformable vesicles}

\section{Vesicle size and zeta potential}

The formulated vesicles $(0.1 \mathrm{ml})$ were diluted (100 times) with phosphate buffer ( $\mathrm{pH}$ 6.8) for vesicle size and zeta potential analysis. Each formulation sample $(0.1 \mathrm{ml})$ was diluted (100 times) with phosphate buffer ( $\mathrm{pH} 6.8$ ), and the size analysis was performed at 25 ${ }^{\circ} \mathrm{C}$ with an angle of detection of $90^{\circ} \mathrm{C}$. The mean vesicle size and zeta potential were evaluated using a Particle size analyzer and Zeta sizer (Horriba Instruments Ltd). The optimized formulation was characterized by a zeta potential value to reveal the stability of the formulation [18].

\section{Determination of entrapment efficiency}

For estimation of the entrapped drug in the prepared vesicle formulation, the unentrapped drug is separated by cooling centrifugation $(7000 \times \mathrm{gm})$ at $4{ }^{\circ} \mathrm{C}$ using a cooling centrifuge for 60 min. Then washing through $40 \mathrm{ml}$ PBS (pH 7.4). The supernatant is collected and the quantity of the unentrapped drug was estimated by UV spectrophotometer at $271 \mathrm{~nm}$, formula for calculating $\%$ entrapment efficiency is as follows [19].

\section{$\%$ Entrapment efficiency}

$$
\begin{aligned}
& =\text { Total amount of drug added } \\
& \text { - Amount of unentrapped drug } \\
& \text { /Drug added x } 100
\end{aligned}
$$

\section{RESULTS AND DISCUSSION}

\section{Optimization of process parameters variables}

During experimental optimization, it is seen that lower speeds of instruments increase the time of contact of the film with the hot water used for the formation of a film, at higher speeds, sufficient time was not available for the lipid to form film and liquid to gel transitions of the lipid is difficult to happen this improper distribution of heat leads to the formation of the uneven film. The hydration of vesicles was affected by different temperatures. When the high temperature is selected for the process, the stability and appearance of vesicle film are not good however, at low temperature a thin film formed, which was uniform and translucent in appearance. Hence, the most appropriate temperature is $50^{\circ} \mathrm{C}$. Finally, it was observed that at $50^{\circ} \mathrm{C}$ temperature and $80 \mathrm{rpm}$ a thin film formed, which was uniform, good in appearance with good stability.

Table 1: Effect of rotation speed and temperature for hydration on the trial batches

\begin{tabular}{llll}
\hline Formulation & $\left(\mathbf{X}_{\mathbf{1}}\right)$ temp $\left({ }^{\circ} \mathbf{C}\right)$ & $\left.\mathbf{X}_{\mathbf{2}}\right) \mathbf{R p m}$ & Condition of formed film* \\
\hline Trial 1 & 50 & 100 & Thin lipid film formed but not rigid and uniform in appearance \\
Trial 2 & 70 & 100 & A bumpy film with entrapped air bubbles \\
Trial 3 & 50 & 80 & The rigid and translucent lipid film was formed with good stability \\
Trial 4 & 60 & 100 & A bumpy film with entrapped air bubbles \\
Trial 5 & 60 & 80 & The rigid film was formed, but less stability \\
Trial 6 & 50 & 60 & Thin lipid film formed but not rigid and uniform in appearance \\
Trial 7 & 70 & 80 & Film not formed \\
Trial 8 & 60 & 60 & Thin lipid film formed but not rigid and uniform in appearance \\
Trial 9 & 80 & 80 & Film not formed \\
\hline
\end{tabular}

*Average of three determinations \pm standard deviation 
Statistical analysis of the data and optimization (Transethosomes)

The responses were fitted into various mathematical models using Design-Expert Version 12 software. The best fit model was decided based on the high values of multiple correlation coefficient $\left(\mathrm{R}^{2}\right)$, adjusted $\mathrm{R}^{2}$, and predicted $\mathrm{R}^{2}$ and low values of standard deviation (SD),. To evaluate the effect of variables on each response, the responses were analyzed to multiple linear regression analysis to generate a second-order quadratic polynomial equation.

Table 2: Experimental runs, independent variables, and measured response of $3^{3}$ full factorial experimental design for transethosomes

\begin{tabular}{|c|c|c|c|c|c|c|}
\hline & $\mathbf{X}_{1}$ & $\mathbf{X}_{2}$ & $\mathbf{X}_{3}$ & $\mathbf{Y}_{1}$ & $Y_{2}$ & \\
\hline Run & $\begin{array}{l}\text { A: Phosphatidylcholine } \\
\text { (mg) }\end{array}$ & B: Ethanol (\%) & C: Span 80 (mg) & Vesicle size* $(\mathrm{nm})$ & $\begin{array}{l}\text { Entrapment } \\
\text { efficiency* (\%) }\end{array}$ & $\begin{array}{l}\text { Zeta potential* } \\
(\mathrm{mV})\end{array}$ \\
\hline 1 & 70 & 40 & 20 & $139.76 \pm 2.25$ & $55.23 \pm 0.23$ & $-20 \pm 0.31$ \\
\hline 2 & 90 & 30 & 20 & $77.23 \pm 4.01$ & $73.82 \pm 1.24$ & $-30 \pm 1.55$ \\
\hline 3 & 70 & 30 & 15 & $112.21 \pm 3.54$ & $79.25 \pm 0.58$ & $-35 \pm 0.66$ \\
\hline 4 & 110 & 30 & 25 & $101.11 \pm 1.48$ & $75.13 \pm 0.78$ & $-32 \pm 0.62$ \\
\hline 5 & 70 & 30 & 25 & $63.21 \pm 2.34$ & $57.39 \pm 2.10$ & $-24 \pm 0.85$ \\
\hline 6 & 70 & 20 & 20 & $110.21 \pm 4.21$ & $72.34 \pm 3.01$ & $-31 \pm 1.58$ \\
\hline 7 & 90 & 40 & 15 & $199.36 \pm 1.52$ & $70.21 \pm 2.01$ & $-30 \pm 1.27$ \\
\hline 8 & 90 & 30 & 20 & $77.21 \pm 3.21$ & $74.42 \pm 0.98$ & $-28 \pm 0.98$ \\
\hline 9 & 90 & 40 & 25 & $126.81 \pm 1.56$ & $58.87 \pm 0.65$ & $-25 \pm 0.81$ \\
\hline 10 & 90 & 30 & 20 & $76.23 \pm 2.56$ & $73.82 \pm 0.47$ & $-30 \pm 0.84$ \\
\hline 11 & 110 & 40 & 20 & $170.12 \pm 1.56$ & $63.74 \pm 1.54$ & $-25 \pm 1.75$ \\
\hline 12 & 90 & 20 & 25 & $132.21 \pm 2.58$ & $73.41 \pm 0.75$ & $-27 \pm 0.36$ \\
\hline 13 & 110 & 20 & 20 & $145.32 \pm 0.31$ & $88.62 \pm 0.35$ & $-31 \pm 0.87$ \\
\hline 14 & 110 & 30 & 15 & $140.58 \pm 0.21$ & $85.81 \pm 0.81$ & $-29 \pm 1.25$ \\
\hline 15 & 90 & 20 & 15 & $145.86 \pm 1.28$ & $96.14 \pm 0.63$ & $-34 \pm 1.66$ \\
\hline
\end{tabular}

*Average of three determinations \pm standard Deviation

\section{Vesicle size analysis}

Vesicle size plays an important role in skin permeation. Smaller vesicular size $(200 \mathrm{~nm})$ facilitates the deformable vesicles to pass through the small pores of the skin leading to more enhanced skin permeation. The average vesicle size of all 15 experimental runs is $121.62 \mathrm{~nm}$, with values lying between the minimum and maximum of $63.21 \pm 2.34 \mathrm{~nm}$ (Formulation 5) and 199.36 $\pm 1.52 \mathrm{~nm}$ (Formulation 7), respectively.

\section{Vesicle size second-order quadratic polynomial equation}

$$
\begin{aligned}
Y_{1}=76.89+16.47 X_{1} & +12.80 X_{2}-21.83 X_{3}-1.19 X_{1} X_{2} \\
& +2.38 X_{1} X_{3}-14.72 X_{2} X_{3}+8.84 X_{1}^{2}+55.62 X_{2}^{2} \\
& +18.55 X_{3}^{2}
\end{aligned}
$$

Where $\mathrm{Y}_{1}$ is Response 1: Vesicle size $(\mathrm{nm}), \mathrm{X}_{1}$ is Phosphatidylcholine $(\mathrm{mg}), \mathrm{X}_{2}$ is Ethanol (\%), $\mathrm{X}_{3}$ is Span $80(\mathrm{mg})$

As per the Quadratic second-order polynomial equation, the phosphatidylcholine is showing a positive effect and the surfactant is exhibiting a negative effect on vesicle size. It was observed that the vesicle size of transethosomes was increased with an increase in the concentration of phosphatidylcholine. The result shows that vesicle size was increased from $63.21 \pm 2.34 \mathrm{~nm}$ (formulation 5) to $170.12 \pm 1.56 \mathrm{~nm}$ (formulation 11) when phosphatidylcholine was increased from $70 \mathrm{mg}$ (formulation 5) to $110 \mathrm{mg}$ (formulation 11). Surfactant also plays a very important role in vesicle size as edge activator. The result shows that Span 80 presented an inverse effect on vesicle size. Vesicle size of prepared transethosomes was decreased on increasing the Span 80 from $15 \mathrm{mg}$ to $25 \mathrm{mg}$. Formulation 7 having Span $8015 \mathrm{mg}$ presented vesicles size of $199.36 \pm 1.52 \mathrm{~nm}$ while formulation 5 presented vesicles size of $63.21 \pm 2.34 \mathrm{~nm}$ which comprised of $25 \mathrm{mg}$ of Span 80. Similarly, formulation 15 (Span 80, 15 $\mathrm{mg}$ ) presented vesicles size of $145.86 \pm 1.28 \mathrm{~nm}$ while formulation 4 (Span 80, $25 \mathrm{mg}$ ) exhibited vesicles size of 101.11 $\pm 1.48 \mathrm{~nm}[20,21]$.

\section{Entrapment efficiency analysis}

The entrapment efficiency of all the formulation was lying in between a minimum and maximum value of (Formulation1) 55.23and (Formulation 15) $96.14 \pm 0.63 \%$ with the average value of $73.21 \%$

Entrapment efficiency second-order quadratic polynomial equation

$\mathrm{Y}_{2}=74.02+6.147 \mathrm{X}_{1}-10.31 \mathrm{X}_{2}-8.33 \mathrm{X}_{3}-1.94 \mathrm{X}_{1} \mathrm{X}_{2}+2.79 \mathrm{X}_{1} \mathrm{X}_{3}$ $+2.85 \mathrm{X}_{2} \mathrm{X}_{3}-2.15 \mathrm{X}_{1}^{2}-1.89 \mathrm{X}_{2}^{2}+2.52 \mathrm{X}_{3}^{2}$
Where $\mathrm{Y}_{2}$ is Response 2: Entrapment efficiency (\%), $\mathrm{X}_{1}$ is Phosphatidylcholine (mg), $\mathrm{X}_{2}$ is Ethanol (\%) and $\mathrm{X}_{3}$ is Span 80 (mg)

A positive value before a factor in the response equation denotes a positive impact that favors optimization, while a negative value indicates a negative impact between the factor and the response. Results show that the concentration of phosphatidylcholine is showing a positive effect on entrapment efficiency while the other two variables ethanol and Span 80 were found to exhibiting a negative effect on the entrapment efficiency of the drug. the concentration of phosphatidylcholine was showing a positive effect. Formulation 13 containing $110 \mathrm{mg}$ of Phosphatidylcholine had exhibited entrapment efficiency of $88.62 \pm 0.35 \%$ on comparison with formulation 1 possessing $80 \mathrm{mg}$ of Phosphatidylcholine showing an entrapment efficiency of $55.23 \pm 0.23 \%$. The gradual increase of entrapment efficiency with an increase in the concentration of phosphatidylcholine is because of the inherent lipophilic character of naproxen sodium as the lipophilic drug would be attracted towards the lipophilic phase and get deposited over there. Ethanol presented an inverse effect on the entrapment efficiency of naproxen sodium in transethosomes. Formulation 15 (ethanol 20\%) presented an entrapment efficiency of $96.14 \pm 0.63 \%$, while formulation 1 having ethanol $40 \%$ presented an entrapment efficiency of $55.23 \pm 0.23 \%$. Similar results were obtained for formulation 13 and 9. Formulation 1 (ethanol 20\%) presented entrapment efficiency of $88.62 \pm 0.35 \%$ and formulation 9 (ethanol $40 \%$ ) presented entrapment efficacy of $58.87 \pm 0.65 \%$. This could be due that at a higher ethanol percentage; vesicles become leakier [22]. Surfactant also plays a very important role in Entrapment efficiency as edge activator. Entrapment efficiency decreased on increasing the concentration of Span 80 Formulation15 (Span 80, 15 $\mathrm{mg}$ ) presented entrapment efficiency of $96.14 \pm 0.63 \%$ while formulation 5 (Span 80, $25 \mathrm{mg}$ ) presented entrapment efficiency of $57.39 \pm 2.10 \%$. Similar results were obtained between formulation 14 (entrapment efficiency $96.14 \pm 0.63 \%$ ) and formulation 9 (entrapment efficiency $58.87 \pm 0.65 \%$ ). The decrease in entrapment efficiency with an increase in the concentration of Span 80 is because of the coexistence of micelle structure with vesicles in the formulation; micelles usually exhibit low entrapment efficiency as compared to vesicles [23].

\section{Zeta potential measurement}


Zeta potential of prepared transethosomes is in the range of$20 \pm 0.31 \mathrm{mv}$ (Formulation 1) and-34 $\pm 1.66 \mathrm{mv}$ (Formulation 15) which suggest relative stability of vesicles with minimum aggregation properties. The highest Zeta potential of vesicles is$34 \pm 1.66 \mathrm{mv}$ (Formulation 15).

Analysis of variance (ANOVA) was applied to determine the statistical significance and the magnitude of the main effects of each variable and their interactions. Counterplots are generated for independent factors. The ANOVA table establishes the sufficiency of the model (i.e., $\mathrm{p}<0.05$ ). The result of the $\mathrm{p}$-value is less than 0.05 for all the response factors signifying that the models are significant. Comparison of the experimental and predicted responses. These data showed that most of the predicted values are nearly similar to the experimental values. These indicated the excellent ability of the experimental design employed for the optimization of transethosomal formulation of Naproxen sodium. The predicted $\mathrm{R}^{2}$ values were in agreement with the adjusted $\mathrm{R}^{2}$ values in all responses. The relationship between the dependent and independent variables was further elucidated using 2D contour plots and 3D response surface plots. Desirability and graphical optimization technique were utilized for generating formulation with the desired responses. The 3D response surface plots are useful to understand the effect of interactions between the factors on a single response.

\section{ANOVA for vesicle size (Response 1)}

The result of P-values is 0.0001 (Significant) the Model F-value is 1811.82 showing that the model is significant. P-values less than 0.05 confirm the significance of the model. Model is not significant when values are greater than 0.1000 . The Predicted $R^{2}$ of 0.9955 agrees with the Adjusted $R^{2}$ of 0.9991 , the difference is less than 0.2 .

\section{ANOVA for entrapment efficiency (\%) (Response 2)}

The result of P-values is 0.0001 (Significant) the model F-value is 1202.04 showing that the model is significant. P-values less than 0.05 confirm the significancy of the model. Model is not significant when values are greater than 0.1000 . The Predicted $R^{2}$ of 0.9944 agrees with the Adjusted $\mathrm{R}^{2}$ of 0.9987 ; the difference is less than 0.2

\section{Selection of the optimum formula}

Further in this study, the point prediction method of Box-Behnken design was utilized for the optimization of transethosomes formulation. The optimized formula desirability function comes higher ie near to 1 confirms the suitability of the formulations. Further, the zeta potential (fig.) value of optimized formulation was found to be-35 mV; the optimized formulation so produced will be further evaluated for vesicles morphology, in vitro release study, skin irritation study, and in vivo study.

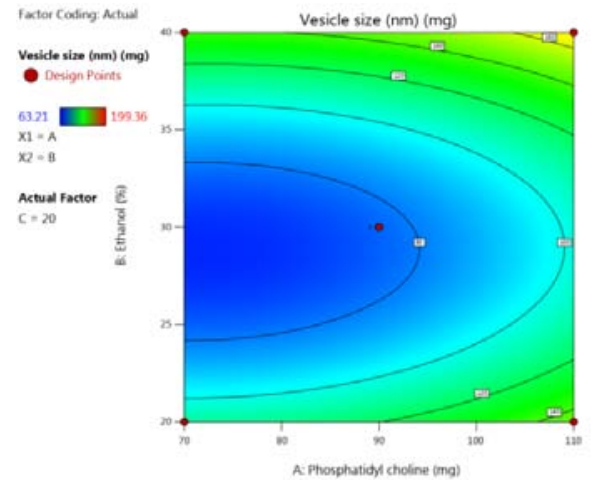

$1 \mathrm{~A}$

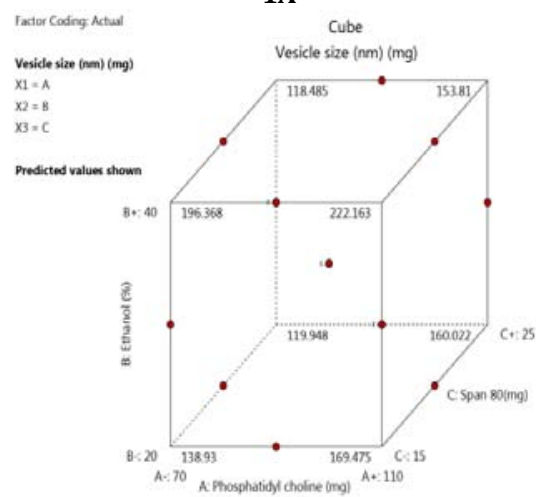

1C

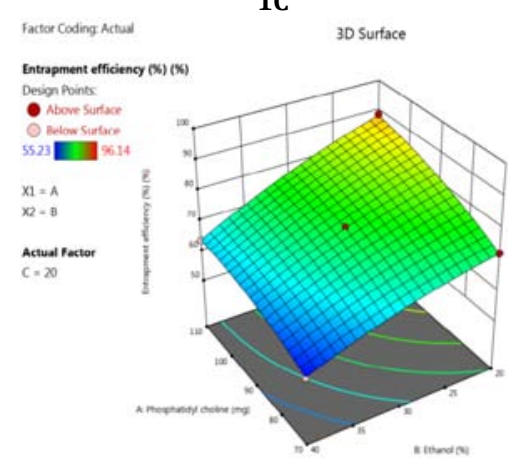

2B

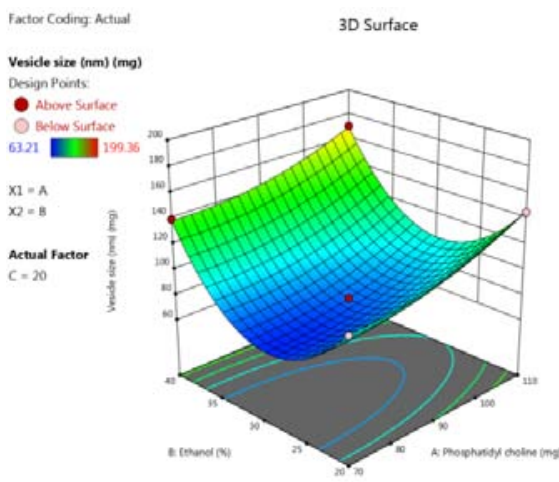

1B

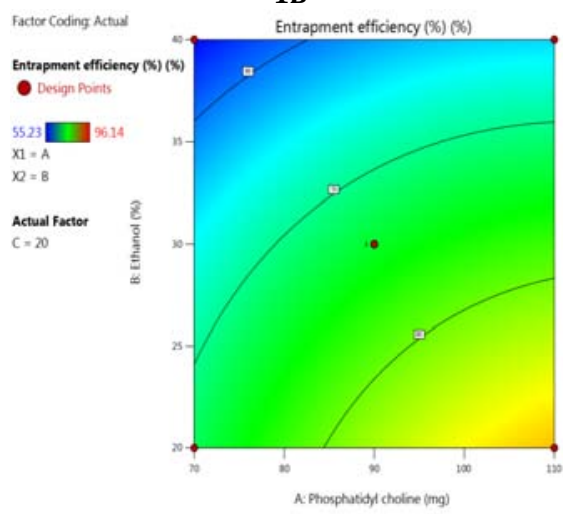

2A

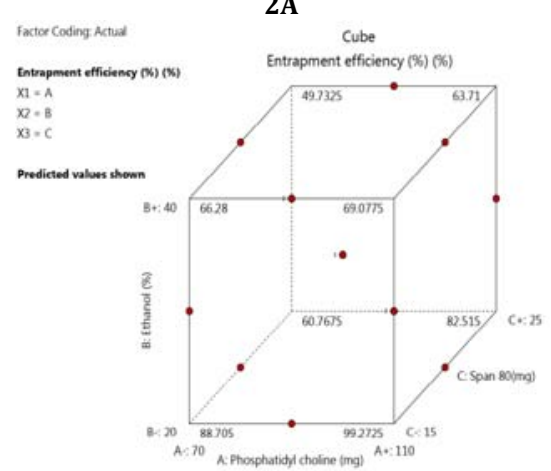

2C 
Fig. 1: Transethosomes factorial design (Box Behnken)

Vesicle size (nm): 2D contour plot (1A). 3D Surface Contour plot (1B). Cube Plot (1C)

Entrapment efficiency (\%): 2D contour plot (2A). 3D Surface Contour plot (2B). Cube Plot (2C)

\section{Statistical analysis of the data and optimization (Transfersomes)}

The responses were fitted into various mathematical models using Design-Expert Version 12 software. The best fit model was decided based on the high values of multiple correlation coefficient $\left(R^{2}\right)$, adjusted $\mathrm{R}^{2}$, and predicted $\mathrm{R}^{2}$ and low values of standard deviation (SD),. To evaluate the effect of variables on each response, the responses were analyzed to multiple linear regression analysis to generate a second-order quadratic polynomial equation.

\section{Vesicle size analysis}

The average vesicle size of all 15 experimental runs was found to $140.20 \mathrm{~nm}$, with values lying between the minimum and maximum of $114.49 \pm 1.54 \mathrm{~nm}$ (Formulation 2) $\mathrm{nm}$ and $174.65 \pm 0.89 \mathrm{~nm}$ (Formulation 10), respectively.

Table 3: Experimental value obtained through point prediction method

\begin{tabular}{lllll}
\hline Composition & Optimized level & Response & Predicted value & Experimental value* $^{*}$ \\
\hline Phosphatidylcholine $(\mathrm{mg})$ & 90.07 & Vesicle size $(\mathrm{nm})$ & 103.19 & 102.91 \\
Ethanol (\%) & 25.07 & Entrapment efficiency & 86.62 & 86.97 \\
Span 80(mg) & 16.53 & $(\%)$ & & \\
\hline
\end{tabular}

Table 4: Experimental runs, independent variables, and measured response of $3^{3}$ full factorial experimental design for transfersomes

\begin{tabular}{lllllll}
\hline & $\mathbf{X}_{\mathbf{1}}$ & $\mathbf{X}_{\mathbf{2}}$ & $\mathbf{X}_{\mathbf{3}}$ & $\mathbf{Y}_{\mathbf{1}}$ & $\mathbf{Y}_{\mathbf{2}}$ & \\
\hline Run & A: Naproxen sodium & $\mathbf{B}$ : Phosphati dylcholine & $\mathbf{C}$ : Span $\mathbf{8 0}$ & Vesicle size $^{*}$ & Entrapment efficiency* & Zeta potential* $^{*}$ \\
\hline & $\mathbf{m g}$ & $\mathbf{m g}$ & $\mathbf{M g}$ & $\mathbf{N m}$ & $\mathbf{\%}$ & $-32 \pm 0.59$ \\
& $\mathbf{1}$ & 80 & 24 & $146.38 \pm 0.23$ & $78.06 \pm 0.11$ & $-31 \pm 1.65$ \\
2 & 35 & 60 & 30 & $114.49 \pm 1.54$ & $75.92 \pm 1.53$ & $-23 \pm 1.51$ \\
3 & 35 & 60 & 18 & $136.54 \pm 0.58$ & $59.17 \pm 1.25$ & $-28 \pm 0.88$ \\
4 & 20 & 60 & 24 & $134.21 \pm 1.56$ & $68.73 \pm 1.56$ & $-36 \pm 1.81$ \\
5 & 20 & 80 & 30 & $123.32 \pm 1.11$ & $89.66 \pm 0.89$ & $-23 \pm 1.11$ \\
6 & 50 & 80 & 18 & $148.67 \pm 2.54$ & $51.46 \pm 1.65$ & $-30 \pm 0.52$ \\
7 & 20 & 100 & 24 & $139.34 \pm 0.78$ & $77.23 \pm 0.23$ & $-31 \pm 1.63$ \\
8 & 35 & 80 & 24 & $146.38 \pm 0.56$ & $74.31 \pm 1.65$ & $-32 \pm 0.41$ \\
9 & 35 & 80 & 24 & $146.38 \pm 2.10$ & $76.48 \pm 0.45$ & $-22 \pm 0.54$ \\
10 & 20 & 80 & 18 & $174.65 \pm 0.89$ & $51.13 \pm 0.82$ & $-37 \pm 1.65$ \\
11 & 35 & 100 & 30 & $119.23 \pm 0.65$ & $89.96 \pm 1.11$ & $-29 \pm 1.55$ \\
12 & 50 & 100 & 24 & $144.23 \pm 0.62$ & $67.64 \pm 1.53$ & $-23 \pm 0.99$ \\
13 & 50 & 60 & 24 & $126.16 \pm 0.35$ & $56.34 \pm 0.45$ & $-29 \pm 0.67$ \\
14 & 50 & 80 & 30 & $135.33 \pm 1.52$ & $70.82 \pm 2.54$ & $-25 \pm 1.77$ \\
15 & 35 & 100 & 18 & $167.77 \pm 0.45$ & $53.46 \pm 0.89$ & \\
\hline
\end{tabular}

*Average of three determinations \pm standard Deviation

\section{Vesicle size quadratic second-order polynomial equation}

$$
\begin{array}{rl}
Y_{1}=146.38-2.141 & 25 X_{1}+7.39625 X_{2}-16.9075 X_{3}+3.235 X_{1} X_{2} \\
& +9.4975 X_{1} X_{3}-6.6225 X_{2} X_{3}+0.295 X_{1}^{2} \\
& -10.69 X_{2}^{2}-1.1825 X_{3}^{2}
\end{array}
$$

Where $Y_{1}$ is Response 1 : Vesicle size $(n m), X_{1}$ is Naproxen sodium ( $\mathrm{mg}$ ), $\mathrm{X}_{2}$ is Phosphatidylcholine $\mathrm{mg}$ ), $\mathrm{X}_{3}$ is Span $80(\mathrm{mg})$

As per the second-order quadratic polynomial equation, the phosphatidylcholine is showing a positive effect on vesicle size, and surfactant and drug concentration were exhibiting a negative effect on vesicle size. The result showed that the vesicle size of transfersomes was increased with an increase in the concentration of phosphatidylcholine formulation 15 with phosphatidylcholine $(100 \mathrm{mg}$ ) has a high vesicle size of $167.77 \pm 0.45$ also formulation 10 with phosphatidylcholine $(80 \mathrm{mg})$ has a high vesicle size of $174.65 \pm 0.89$. Surfactant also plays a very important role in vesicle size as edge activator. It was observed that Span 80 presented an inverse effect on vesicle size formulation 10 with span $80(18 \mathrm{mg})$ has a high vesicle size of $174.65 \pm 0.89$ also formulation 15 with span 80 (18 mg) has a high vesicle size of $167.77 \pm 0.45$. As the drug concentration reaches the lowest vesicle size becomes highest for example formulation 10 with Naproxen sodium $(20 \mathrm{mg})$ has a high vesicle size of $174.65 \pm 0.89$.

The surfactants destabilize vesicular bilayer reduces interfacial tension hence enhances bilayer elasticity, hence leads to a decreased particle size. For skin delivery, small particle size is of importance for skin penetration and drug deposition [24]. New research shows a mechanistic picture of vesicle solubilization formation and concluded that surfactant-containing bilayers, at certain lipid/surfactant ratio, spontaneously un/curve and open or close to optimizing edge tension [25]. In transfersomes incorporation of edge activators in low concentration results in vesicle size growth [26].

\section{Entrapment efficiency analysis}

The entrapment efficiency of all the formulation found in between a minimum and maximum value of (Formulation 10 ) $51.13 \pm 0.82 \%$ and (Formulation 11) $89.96 \pm 1.11 \%$ with the average value of $69.36 \%$.

Entrapment efficiency second-order quadratic polynomial equation

$$
\begin{aligned}
& Y_{2}=76.2833-5.06125 X_{1}+3.51625 X_{2}+13.8925 X_{3}+0.7 X_{1} X_{2} \\
&-4.7925 X_{1} X_{3}+4.9375 X_{2} X_{3}-6.32917 X_{1}^{2} \\
&-2.46917 X_{2}^{2}-4.18667 X_{3}^{2}
\end{aligned}
$$

Where $Y_{2}$ is Response 2: Entrapment efficiency (\%), $X_{1}$ is Naproxen sodium (mg), $X_{2}$ is Phosphatidylcholine (mg), $X_{3}$ is Span $80(\mathrm{mg})$.

Results showed that the concentration of phosphatidylcholine and span 80 were showing a positive effect on entrapment efficiency, while the naproxen sodium was found to exhibiting a negative effect on the entrapment efficiency of the drug. The result shows that the entrapment efficiency of transfersomes was increased with an increase in the concentration of phosphatidylcholine formulation 11 with phosphatidylcholine (100 mg) shows high entrapment efficiency of $89.96 \pm 1.11$. Surfactant also plays a very important role 
in Entrapment efficiency as edge activator. Entrapment efficiency increases on increasing the concentration of Span 80 Formulation11 (Span $80 \quad 30 \mathrm{mg}$ ) presented high entrapment efficiency of $89.96 \pm 1.11 \%$ while formulation 5 (Span $8030 \mathrm{mg}$ ) presented high entrapment efficiency of $89.66 \pm 0.89 \%$. As Naproxen sodium concentration decreases entrapment efficiency increases. As the drug concentration reaches the lowest entrapment efficiency becomes highest for example, formulation 5 with Naproxen sodium $(20 \mathrm{mg}$ ) has a high Entrapment efficiency of $89.66 \pm 0.89 \%$

\section{Zeta potential measurement}

Zeta potential values of prepared transfersomes is in the range of$22 \pm 0.54 \mathrm{mv}$ (Formulation 10) and $-37 \pm 1.6554 \mathrm{mv}$ (Formulation 11) which suggest relative stability of vesicles with minimum aggregation properties Highest Zeta potential of vesicles is$37 \pm 1.6554 \mathrm{mv}$ (Formulation 11).

\section{Analysis of variance (ANOVA)}

The ANOVA table confirms the adequacy of the model (i.e., $p<0.05$ ). The p-value is less than 0.05 for all the response factors indicating that the models are significant. These data showed that most of the predicted values were close to the experimental values. These indicated the excellent prognostic ability of the experimental design employed for the optimization of transfersomal formulation of Naproxen sodium. The predicted $\mathrm{R}^{2}$ values were in reasonable agreement with the adjusted $\mathrm{R}^{2}$ values in all responses.

\section{ANOVA for vesicle size (Response 1)}

The result of P-values is 0.0002 (Significant) the Model F-value is 53.15 showing that the model is significant. P-values less than 0.05 confirm the significancy of the model. Model is not significant when values are greater than 0.1000 . The Predicted $R^{2}$ of 0.8345 agrees with the Adjusted $\mathrm{R}^{2}$ of 0.9710 ., the difference is less than 0.2. [27, 28].

\section{ANOVA for entrapment efficiency (\%) (Response 2)}

The result of P-values is 0.0003 (Significant) the Model F-value is 45.06 showing that the model is significant. P-values less than 0.05 confirm the significancy of the model. Model is not significant when values are greater than 0.1000 . The Predicted $R^{2}$ of 0.8479 agrees with the Adjusted $R^{2}$ of 0.9659 , the difference is less than 0.2

\section{Selection of the optimum formula}

Further in this study, the point prediction method of Box-Behnken design was utilized for the optimization of transfersomes formulation. The optimized formula desirability function comes higher ie near to 1 confirms the suitability of the formulations. Further, the zeta potential value of optimized formulation was found-37 mV, respectively. The optimized formulation so produced will be further evaluated for vesicle morphology, in vitro release study, skin irritation study, and in vivo study.

Table 5: Experimental value obtained through point prediction method

\begin{tabular}{lllll}
\hline Composition & Optimized level & Response & Predicted value & Experimental value* \\
\hline Naproxen sodium (mg) & 20.74 & Vesicle size (nm) & 114.33 & 114.91 \\
Phosphatidylcholine (mg) & 60.70 & Entrapment efficiency (\%) & 79.69 & 80.11 \\
Span 80(mg) & 29.86 & & & \\
\hline
\end{tabular}

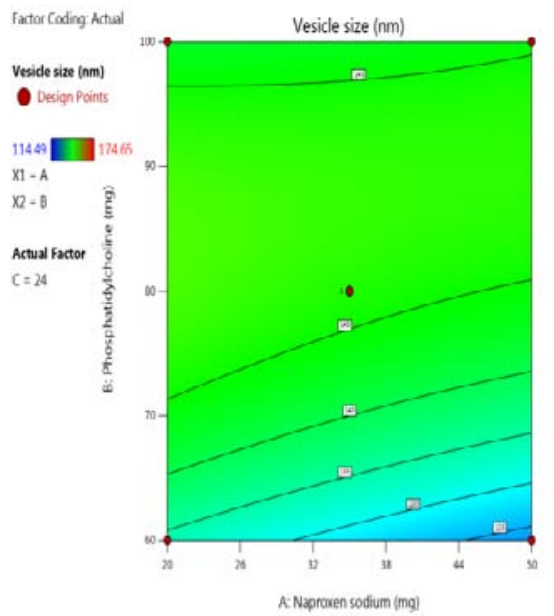

$1 \mathrm{~A}$

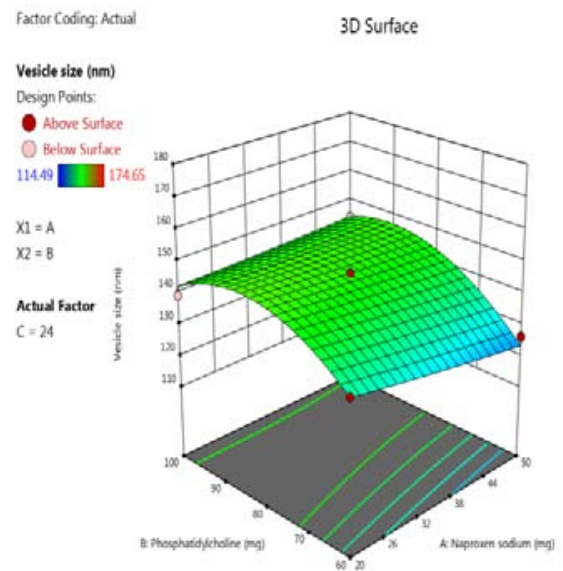

1B 


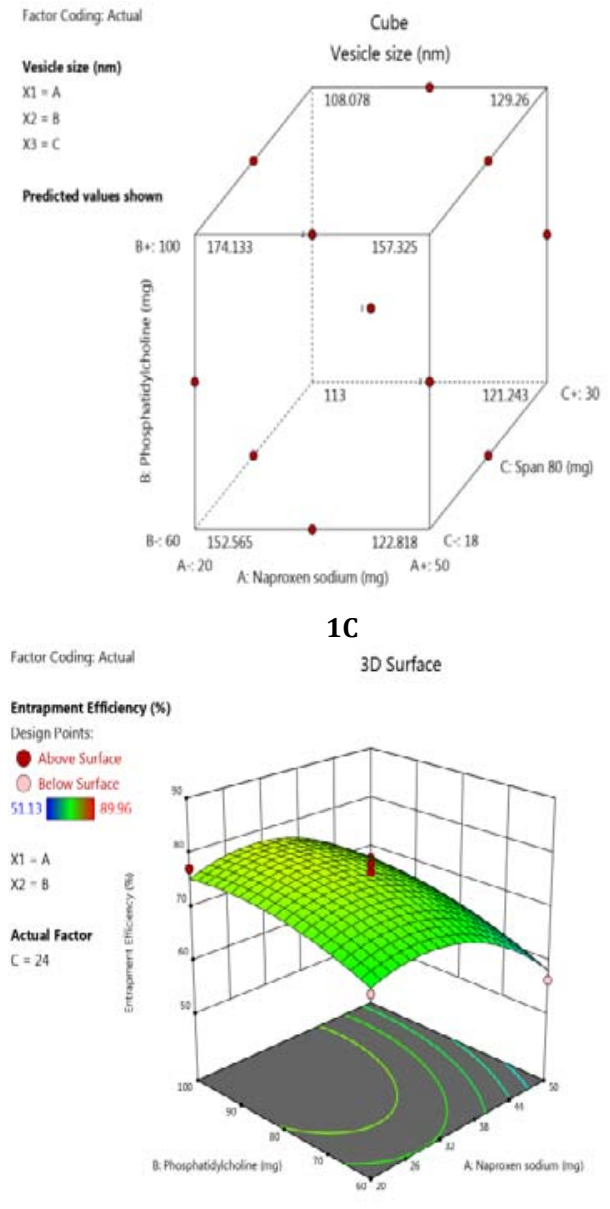

$2 B$

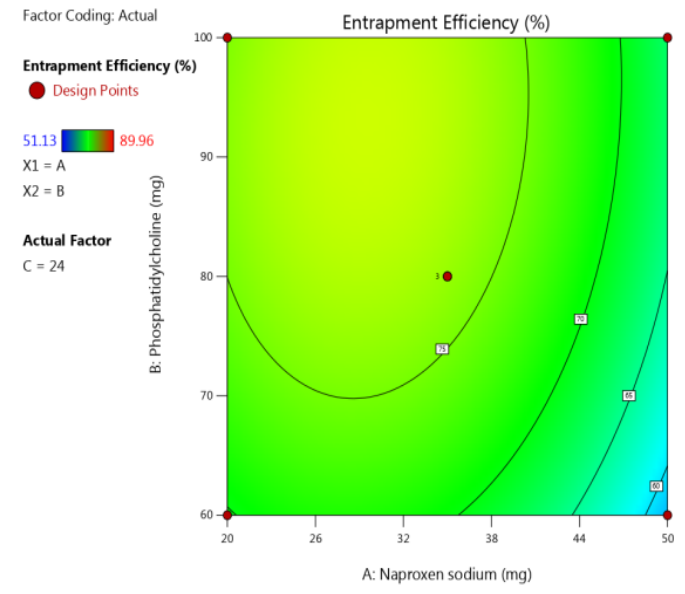

2A

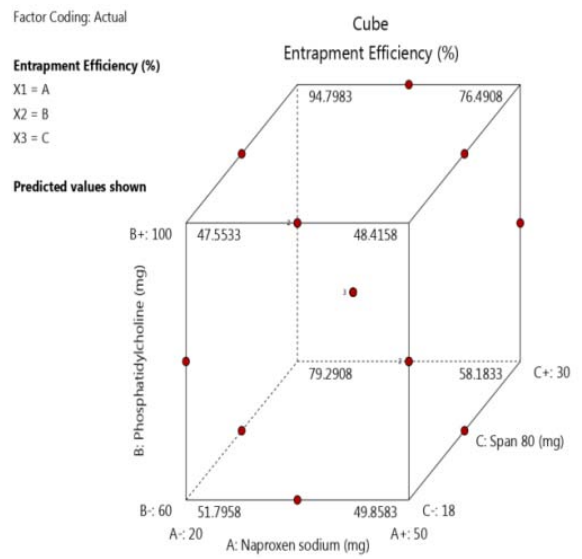

2C

Fig. 2: Transfersomes factorial design (Box Behnken)

Vesicle size (nm): 2D contour plot (1A). 3D Surface Contour plot (1B). Cube Plot (1C)

Entrapment efficiency (\%):2D contour plot (2A). 3D Surface Contour plot (2B). Cube Plot (2C)

\section{CONCLUSION}

Present study utilizes a factorial design (Box Behnken) for the preparation of naproxen sodium transfersomes and transethosomes with desired characteristic responses. The factorial design describes the relationship between an experimental response and a set of input variables. Optimization of vesicles signifies a point prediction model for the selection of variable ranges to achieve the expected desired outcome and responses. Within a small experimental trial setup, the desired responses can be achieved by a systematic experimental formulation approach for establishing a mathematical trend in the experimental design. In our study, optimized formulations show minimum vesicle size, good entrapment efficiency, and optimum zeta potential, which justify the usefulness of drug carrier for dermal delivery of naproxen sodium.

\section{FUNDING}

Nil

\section{AUTHORS CONTRIBUTIONS}

All the authors have contributed equally.

\section{CONFLICT OF INTERESTS}

The authors declare no conflict of interest in this work.

\section{REFERENCES}

1. Benson H. Transfersomes for transdermal drug delivery. Expert Opinion Drug Delivery 2006;3:727-37.

2. Priya K, Kumar V, Damini V, Eswar K, Reddy KR, Brito Raj S, Sucharitha P. Somes: a review on composition, formulation methods and evaluations of different types of "somes" drug delivery system. Int J Appl Pharm 2020;12:7-18.

3. Pandey P, Pancholi S. Nanocarriers: a novel treatment approach for arthritis. Int J Pharm Sci Res Vol 2013;4:4165-74.

4. Kumavat S, Chaudhari Y, Borole P. Transfersomes: a promising approach for transdermal drug delivery system. Asian J Pharm Sci 2013;3:1-17.

5. Mezei M, Gulasekharam V. Liposomes-a selective drug delivery system for the topical route of administration lotion dosage form. Life Sci 1980;26:1473-7.

6. Verma D, Verma S, Blume G. Particle size of liposomes influences dermal delivery of substances into the skin. Int J Pharm 2003;258:141-51.

7. Manosroi A, Jantrawut P, Manosroi J. Anti-inflammatory activity of gel containing novel elastic niosomes entrapped with diclofenac diethyl ammonium. Int J Pharm 2008;360:156-63.

8. G Cevc. Transfersomes, liposomes, and other lipid suspensions on the skin: permeation enhancement, vesicle penetration, and transdermal drug delivery. Crit Rev Ther Drug Carrier Syst 1996;13:257-388.

9. Maghraby G, Williams A, Barry A. Interactions of surfactants (edge activators) and skin penetration enhancers with liposomes. Int J Pharm 2004;276:143-61.

10. E Touitou, M Alkabes, N Dayan. Ethosomes-novel vesicular carriers for enhanced delivery: characterization and skin penetration properties. PharmRes 2000;65:403-18. 
11. Elsayed M, Abdallah O, Naggar V, Khalafallah V. Lipid vesicles for skin delivery of drugs: reviewing three decades of research. Int J Pharm 2007;332:1-16.

12. Chaurasia G, Lariya N. A comparative assessment of vesicular formulations: transfersomes and conventional liposomes loaded ivabradine hydrochloride. Int J Appl Pharm 2020;12:51-5.

13. Shaji J, Vinit M. Novel double-loaded transferosomes: evidence of superior anti-inflammatory efficacy-a comparative study. Int J Curr Pharm 2014;6:16-25.

14. Shaji J, Bajaj R. Formulation development of 5-fluorouracil transethosomes for skin cancer therapy. Int J Pharm 2017;11:453-64.

15. Shaji J, Maria Lal. Preparation, optimization, and evaluation of transferosomal formulation for enhanced transdermal delivery of a cox-2 inhibitor. Int J Pharm Pharm Sci 2014;6:467-77.

16. Vyas P, Vyas P, Raval D, Paghdar P. Development of topical niosomal gel of benzoyl. Nanotechnology 2011. https://doi.org/10.5402/2011/503158.

17. Sara M Soliman, Nevine S Abdelmalak, Omaima N. Novel nonionic surfactant proniosomes for transdermal delivery of lacidipine: optimization using 23 factorial design and in vivo evaluation in rabbits. Drug Delivery 2016;23:1608-22.

18. Mohanty D, Rani M, Haque M. Preparation and evaluation of transdermal-naproxen niosomes: formulation optimization to preclinical anti-inflammatory assessment on the murine model. J Liposome Res 2019;30:1-11.

19. Alima S, Kassem A, Bashaa M, Salama A. Comparative study of liposomes, ethosomes, and transfersomes as carriers for enhancing the transdermal delivery of diflunisal: in vitro and in vivo evaluation. Int J Pharm 2019;30:293-303.
20. Touitou E, Dayan N, Bergelson L. Ethosomes-novel vesicular carriers for enhanced delivery: characterization and skin penetration properties. J Controlled Release 2000;65:403-18.

21. Dubey V, Mishra D, Dutta T. Dermal and transdermal delivery of an anti-psoriatic agent via ethanolic liposomes. J Controlled Release 2007;123:148-54.

22. Ahad A, Aqil M, Kohli K. Enhanced transdermal delivery of an anti-hypertensive agent via nanoethosomes: statistical optimization characterization and pharmacokinetic assessment. Int J Pharm 2013;443:26-38.

23. El Zaafarany GM, Awad GA, Holayel SM. Role of edge activators and surface charge in developing ultra deformable vesicles with enhanced skin delivery. Int J Pharm 2010;397:164-72.

24. Chen G, D Li, Y Jin, Zhang W, Teng L. Deformable liposomes by reverse-phase evaporation method for enhanced skin delivery of (b)-catechin. Drug Dev Ind Pharm 2013;40:260-5.

25. Simoes SI, Marques CM, Cruz ME, Cevc G, Martins M. The effect of cholate on solubilization and permeability of simple and protein-loaded phosphatidylcholine/sodium cholate mixed aggregates designed to mediate transdermal delivery of macromolecules. Eur J Pharm Biopharm 2004;58:509-19.

26. Van den Bergh BA, Wertz PW, Junginger HE, Bouwstra JA. The elasticity of vesicles assessed by electron spin resonance, electron microscopy, and extrusion measurements. Int J Pharm 2001;1:13-24.

27. Armatazaka Z, Sulaiman TN, Zulkarnain AK. Optimization and characterization of PEG-PCL-PEG triblock copolymer as a carrier of drug-using full factorial design. Int J Curr Pharm Res 2020;11:65-71.

28. Chuo W, Kuang Y, Huang YT, Shan C. Statistical optimization and stability study of the quercetin-loaded microemulsion. Int J Pharm Pharm Sci 2020;13:13-25. 